\title{
Le jeu de rôle sur table : une forme littéraire intercréative de la fiction?
}

Coralie David

\section{(2) OpenEdition \\ 12 Journals}

Édition électronique

URL : http://journals.openedition.org/sdj/682

DOI : $10.4000 /$ sdj. 682

ISSN : 2269-2657

\section{Éditeur}

Laboratoire EXPERICE - Centre de Recherche Interuniversitaire Expérience Ressources Culturelles Education

\section{Référence électronique}

Coralie David, « Le jeu de rôle sur table : une forme littéraire intercréative de la fiction ? », Sciences du jeu [En ligne], 6 | 2016, mis en ligne le 13 octobre 2016, consulté le 20 avril 2019. URL : http:// journals.openedition.org/sdj/682 ; DOI : 10.4000/sdj.682

Ce document a été généré automatiquement le 20 avril 2019.

Tous droits réservés 


\title{
Le jeu de rôle sur table : une forme littéraire intercréative de la fiction?
}

\author{
Coralie David
}

\section{RolePlaying Game : play et game, une tension créatrice}

1 Depuis son apparition en 1974 avec Dungeons \& Dragons, le jeu de rôle (JdR), traduction forcément incomplète du terme roleplaying game en anglais, porte en lui la polysémie bipartite du jeu. Donald Winnicott (1975) et Jean Duvignaud (1980, p. 14) distinguent le game qui organise le déroulement selon un processus précis, du play qui relève davantage de l'espace libre que l'on investit. Cette distinction entre play et game, liberté et règles, est cruciale lorsqu'il s'agit d'envisager les JdR. Comme tension créatrice, elle apparaît également assez tôt dans les théories de la fiction, en tant que processus de création: Wayne C. Booth (1961), notamment, pose la fiction comme un procédé double, entre imagination (que l'on pourrait rapporter au play, à la matière créatrice jaillissante et incontrôlée) et mise en œuvre (potentiellement assimilée au game, qui viendrait organiser cette matière pour la rendre propre à la réception, intelligible).

Par conséquent, dans le jeu comme dans la fiction, le play est une manifestation intérieure de l'individu, une expression créatrice, alors que le game est essentiellement une force de l'extérieur : il vient, en ordonnant le chaos créateur, permettre la réception, l'échange, en créant un espace imaginaire commun où se jouera la réception. Et si le game n'était qu'une socialisation, une mise en collectivité du play individuel ? Et si le game n'était que l'évolution sociale et adulte du play? C'est ce que semble penser Roger Caillois (1957), quand il évoque la tension entre ludus et paidia. La fonction du game serait dès lors de générer des règles permettant de partager un espace sémantique commun, d'organiser collectivement une activité créatrice. C'est précisément la fonction du système de JdR sur table, que nous définissons comme l'ensemble des dispositifs qui rend la fiction 
interactive et intercréative. Son rôle est d'intégrer harmonieusement l'apport créatif des différents joueurs. Le game organise le play de différents individus en un ensemble cohérent pour aboutir à une création fictionnelle de groupe.

3 Le système de $\mathrm{JdR}$ n'est donc pas une règle qui réduit la liberté des joueurs, il est un contrat qui explicite les « rôles » de chacun dans la création fictionnelle par la narration orale. Dès lors, le système de JdR se pose comme le garant de la légaliberté de Colas Duflo (1997, p. 221): «Le contrat ludique instaure le règne de la légaliberté, c'est l'acte par lequel chacun se soumet aux règles du jeu, créant par là le monde ludique. ». Dans le cas $\mathrm{du} \mathrm{JdR}$, cette légaliberté prend la forme d'une garantie de répartition de l'autorité générative (qui érige les éléments diégétiques composant l'univers) et résolutive (décider de l'issue d'une situation incertaine) entre les joueurs, dont la proportion varie selon les types de jeu'.

4 C'est la raison pour laquelle dès la première édition de Dungeons \& Dragons, il est conseillé aux joueurs de créer leurs propres règles, si les personnages se retrouvent dans une situation imprévue. Lorsque le play est amené à les faire sortir du cadre du game, c'est aux joueurs d'étendre le game en fonction. De fait, le game devient également un outil pour se modifier lui-même. En un sens, le play a autorité sur le game.

\section{L'intercréativité, spécificité des JdR sur table}

5 La manière unique dont les JdR vont articuler play et game est la base de l'intercréativité. Celle-ci est rendue possible au terme de trois étapes, qui commencent dès la création d'un JdR jusqu'aux parties qu'il permet de jouer. Nous prenons aussi bien en compte les JdR sans meneur de jeu (MJ) que ceux dits traditionnels comprenant un MJ, puisque ces jeux n'accomplissent finalement qu'une redistribution des fonctions créatives du MJ autour de la table. De surcroît, y compris dans les JdR avec MJ, ces fonctions peuvent varier en termes d'autorité sur la fiction et montrent de grandes variations d'un jeu à l'autre (voir note 1).

6 La première est la disruption du bloc fictionnel (une unité contenant en un seul ensemble un univers, des personnages et un récit, comme un roman ou un film, ou un genre fictionnel comme la science-fiction, ou des scènes archétypes). Elle consiste à identifier et à désolidariser les éléments qui composent les mécanismes et les codes d'un bloc fictionnel.

7 Par exemple, la première édition de Dungeons \& Dragons (D\&D) revendique comme source la littérature fantasy. Les auteurs du jeu, Dave Arneson et Gary Gygax, identifient les codes du genre et les décomposent pour en faire des mécanismes ludiques : chaque personnage de la Terre du Milieu de J. R. R. Tolkien est une unité formée de caractéristiques. Une fois désolidarisées de cet ensemble, elles deviennent les classes et les races indispensables pour créer un personnage-joueur (PJ) dans le jeu: Aragorn est, avant que l'on ne découvre sa véritable identité, le chevalier errant des légendes arthuriennes, le rôdeur humain (terme qui deviendra le nom exact d'une classe de personnage de D\&D), les hobbits Pippin et Merry sont les stéréotypes des roublards du jeu, Gimli, le nain bourru des montagnes qui se bat à la hache est un guerrier, Legolas est le bel elfe archer et Gandalf le vieux mage sage. La mémorisation des sorts est empruntée à Jack Vance et son cycle The Dying Earth, l'alignement à Michael Moorcock et à Poul Anderson. Ces éléments identifiés, ils sont transformés par les concepteurs en mécanismes ludiques. D’emblée, le 
$\mathrm{JdR}$ emprunte ses codes aux wargames ${ }^{2}$ et à la littérature, ce qui le place à la frontière de ces deux sphères.

8 Cela nous amène à la deuxième étape réalisée par les auteurs de JdR, la systémation, c'està-dire le fait de mettre ces mécanismes à disposition des joueurs, de les rendre utilisables pour qu'ils puissent les manier comme des outils de création de leurs propres éléments fictionnels. Pour reprendre notre exemple, les classes et les races permettent de créer un personnage. Cette mise en système des composants des romans comme mécanismes ludiques les transforme en outils de création qui permettent de rester dans un cadre défini, ici la littérature fantasy classique. Cette systémation permet pourtant de générer des parties dont la fantasy est unique et spécifique à $D \& D$, car chaque jeu réorganise les codes d'une façon qui lui est propre.

La troisième étape est la réorganisation narrative de ce matériau fictionnel que les joueurs créent ensemble. Ce matériau est d'une part fourni par le jeu, et d'autre part créé par les joueurs. C'est pour cette raison que nous parlons d'un média intercréatif : cette intercréativité est démiurgique et narrative : les joueurs intègrent certains éléments déjà connus et en créent de nouveaux, qu'ils réagencent pour créer une nouvelle unité, un nouveau bloc fictionnel qui est leur création. Pour la définir, l'intercréativité permet de co-créer une fiction au lieu de limiter les choix des récepteurs au simple fait de réagir à un contenu qui leur est proposé. En cela elle représente un prolongement de l'interactivité. La réception devient dès lors une étape du processus créatif.

Comme la vidéo a permis l'avènement des deux médias différents que sont le cinéma et le jeu vidéo en organisant différemment le rapport d'interactivité, le langage est également le moyen par lequel la fiction est créée et reçue en JdR et en littérature. Toutefois, le JdR est un média différent car il propose de faire une utilisation intercréative du langage.

11 Si aujourd'hui la sphère littéraire est indissociable de l'écrit, il est intéressant de l'envisager dans une perspective plus large, notamment dans sa dimension orale. Nous proposons ainsi une distinction entre littérature apollinienne, écrite, figée et créée par un auteur et lue par un récepteur dont les statuts et fonctions sont clairement identifiés, et une littérature dionysiaque, autotélique, orale, présentifiée et collectivement générée. Apollinienne et dionysiaque ne sont pas à prendre dans leur sens nietzschéen. Par apollinien, nous voulons souligner le caractère long et réfléchi du travail de l'écrivain avant l'accès au lecteur: améliorations et reformulations stylistiques, construction, anticipation des réactions du « lecteur modèle » (Eco 1979, p. 68), etc., bref l'ensemble des étapes qui mène au récit au sens de Gérard Genette (1972 p. 72) : "signifiant, énoncé, discours ou texte narratif ». Le JdR n'a en effet pas vocation à livrer un récit en tant que « produit fini » pouvant être lu (d'où son statut autotélique), mais une narration, toujours selon la définition de Genette (1972 p. 72) : «L'acte narratif producteur et, par extension, l'ensemble de la situation réelle ou fictive dans laquelle il prend place» Par dionysiaque, nous entendons par conséquent l'aspect spontané, instinctif et immédiat qui caractérise la conversation créatrice de fiction qui émerge lors d'une partie de JdR.

12 Mais pourquoi envisager le JdR comme une forme de littérature? Il ne s'agit pas d'une manière de légitimer son étude, mais bien de s'interroger sur la relation entre jeu et art et la façon dont sont catégorisés l'un ou l'autre: les frontières peuvent être poreuses, et déplacer notre angle de vue permet d'ouvrir des perspectives en termes d'outils d'analyse et d'inscription dans la culture populaire. En l'occurrence, il s'agit de mener une réflexion sur la reconquête de la création fictionnelle par le jeu par des individus qui ne sont $a$ priori pas considérés comme des auteurs. Le JdR a ouvert un interstice pour expérimenter 
un autre rapport à la fiction, que nous nous proposons d'étudier ici. Pour cela, il faut garder à l'esprit la distinction que fait Richard Hoggart (1970) entre la culture de masse, produite et imposée, comme la culture attestée, par des élites, dont le seul but est d'être rentable, et la culture populaire qui génère, via ses récepteurs et créateurs, ses propres codes qui ne sont pas seulement imposés par les grands médias.

Nous allons nous attacher à répondre aux questions suivantes : le JdR permettant à ses participants de créer de la fiction grâce au langage verbal (les illustrations et autres "marqueurs ludiques» comme les dés ou les feuilles de personnages n'étant pas indispensables à sa pratique, bien qu'ils constituent des outils précieux pour cette cocréation), quel est son rapport à la littérature ? Comment réorganise-t-il les positions de création et de réception, les rapports entre joueur, auteur et lecteur, étant donné qu'il faut lire un jeu avant d'y faire jouer, au moins pour un des joueurs ? Peut-il être considéré comme une forme de jeu littéraire oral et collectif? Dans un premier temps, nous étudierons le rapport qu'entretiennent littérature et jeu d'une façon générale. Ensuite, nous verrons que le JdR est un processus conversationnel, oral et présentifié qui génère une création fictionnelle collective.

\section{Jeu et littérature, approche théorique d'un rapport ambivalent}

14 Umberto Eco (1979) avait perçu le rapport entre littérature et jeu, auquel nous allons nous consacrer ici, dès les années 1970. Néanmoins, encore en 1986, Michel Picard (1986, p. 11) relève que « le jeu est le refoulé des études littéraires ». Gageons que le jeu, chargé d'une symbolique puérile, réglé par des mécanismes qui semblent contraignants et pauvres par leur logique combinatoire, et assimilé au pur divertissement et à la culture de masse, peinait à être envisagé comme un espace de réception possible et acceptable pour un art. Ces a priori sont à présent remis en question, et l'exploration de nouveaux territoires à la marge de la littérature et du jeu font l'objet de nombreuses recherches, comme les travaux de la narratologue Marie-Laure Ryan (2003) sur les formes interactives numériques, ceux de Nick Montfort sur le même sujet, qui consacre d'ailleurs son chapitre 3 aux liens séminaux avec Dungeons \& Dragons (Montfort, 2005, p. 65 et suivantes), ou encore ceux de Serge Bouchardon (2008) en Sciences de l'information et de la communication. Nous inscrivons cet article dans ce cheminement.

\section{Étymologie et porosité des diégèses : des racines entrelacées}

D'un point de vue étymologique, littérature et jeu présentent des origines communes :

On appelait jongleur, au Moyen Âge, un musicien exécutant ou un diseur d'œuvres littéraires, établi à son compte. [...] Le jongleur était l'intermédiaire entre l'auteur et le public, comme aujourd'hui l'acteur de théâtre; mais dans une civilisation comportant une grande quantité d'analphabètes, il faisait connaître oralement la littérature lyrique ou narrative (Souriau, 1990, p. 922).

Jongleur vient du latin joculator, dont la racine jocus a donné le terme jeu en français. Par conséquent, on pourrait se risquer à supposer que la dichotomie entre jeu et littérature n'est pas si ancienne. Françoise Lavocat identifie des formes ludiques de réception de la littérature dès le XVII ${ }^{e}$ siècle : 
La figure de Don Quichotte, qui hante un grand nombre de mascarades, de tournois, de ballets de la première moitié du dix-septième siècle, immanquablement associée à toute allusion à la littérature dans la sphère ludique du divertissement de cour dans cette période et au-delà, est un autre indice de la proximité entre le jeu et la notion de fiction (Lavocat, 2005, p. 90).

Et encore en 1689 :

Une sorte de jeu de l'oie, inventé à la cour de Savoie à partir du Roland furieux de l'Arioste, et appelé "Il laberinto de l'Ariosto, Giocoheroico di Cavalieri e Dame». Les joueurs portaient les noms des héros principaux du poème, également représentés par des figurines de cire qu'ils faisaient progresser à coups de dés dans les cases d'un labyrinthe, où étaient figurés les épisodes principaux de l'œuvre (Lavocat, 2005, p. 89).

18 Jeu et littérature ont un pouvoir démiurgique, ils sont créateurs d'univers qu'ils peuvent se partager. Le fait que ces diégèses ${ }^{3}$ deviennent le cadre d'un récit ou celui d'un jeu n'est qu'une question d'usage.

Pour certains, les règles, le ludus logique, seraient susceptibles d'entrer en conflit avec le caractère créateur et chaotique de l'art, qui est supposé s'affranchir de telles contraintes pour laisser s'exprimer originalité et inventivité. Pourtant, en 1674, Boileau n'envisage pas les règles strictes comme des obstacles à la création littéraire, bien au contraire. Elles sont un outil de création qui fonctionne symbiotiquement avec le génie littéraire :

La rime est une esclave et ne doit qu'obéir.

Lorsqu'à la bien chercher d'abord on s'évertue,

L'esprit à la trouver aisément s'habitue ;

$\mathrm{Au}$ joug de la raison sans peine elle fléchit

Et, loin de la gêner, la sert et l'enrichit (Boileau, 1674).

Finalement, la fiction, au-delà de la littérature, est aussi création réglée, et Jean-Marie Schaeffer pose cette caractéristique comme un trait commun avec le jeu : «comme tout jeu, la fiction instaure ses propres règles, ce qui implique une suspension provisoire (et partielle) de celles qui valent en dehors de l'espace ludique » (Schaeffer, 1999, p. 151). À ses yeux les «fictions numériques » représentent une innovation signifiante en cela qu' «elles réconcilient la fiction avec ses origines ludiques.» (Schaeffer, 1999, p. 315). Les genres vidéoludiques que sont le RPG et le MMORPG étant largement inspirés du JdR sur table, il semblerait que cette "réconciliation» entre jeu et art se soit amorcée avant même l'avènement des jeux vidéo.

\section{La réception de la littérature, un acte ludique}

21 Comme moyen d'entrée dans la fiction en général et dans la littérature en particulier, la « feintise ludique» (Schaeffer, 1999, p. 11) est suggérée dès 1979 par Umberto Eco dans Lector in Fabula. Pour lui, le texte est une " machine présuppositionnelle » (Eco, 1979, p. 27) qui prévoit le lecteur et même, exige que celui-ci l'aide à fonctionner en remplissant ses espaces blancs. Comme dans un jeu, le texte présente une dynamique stratégique : "L'auteur prévoit un Lecteur Modèle capable de coopérer à l'actualisation textuelle de la façon dont lui, l'auteur, le pensait; et capable aussi d'agir interprétativement comme lui a agi générativement » (Eco, 1979, p. 68).

Umberto Eco met en exergue trois notions cruciales pour le dispositif ludique d'entrée dans la littérature : l'encyclopédie personnelle, donc la culture fictionnelle et intertextuelle du lecteur, les promenades inférentielles, ces moments où le lecteur a recours aux scénarios 
communs ou intertextuels d'œuvres qu'il a précédemment lues pour tenter d'anticiper certains segments narratifs, et les mondes possibles, qui font que l'acte de lecture, par ces promenades inférentielles qui sont en fait un ensemble de propositions fictionnelles à l'intérieur de la diégèse, crée des mondes possibles coexistants. En lisant, le lecteur configure ces mondes possibles.

Ces trois points sont utiles pour comprendre le lien entre JdR et littérature, puisque les $\mathrm{JdR}$ vont hypertrophier ces trois opérations de réception jusqu'à aboutir à l'intercréativité. De la même manière, Wolgang Iser explique que le lecteur veut " jouer " avec le texte littéraire, dans le sens où il veut se confronter à lui par ses promenades inférentielles, soucieux de vérifier ses pronostics : «la lecture ne devient un plaisir que si la créativité entre en jeu, que si le texte nous offre une chance de mettre nos aptitudes à l'épreuve.» (Iser, 1995, p. 199). Il rappelle qu'un texte est avant tout un dispositif communicationnel, comme l'est l'intercréativité, dont la signification se construit durant l'acte de lecture, ainsi peut-il faire l'objet de réceptions multiples et contient en germe une pluralité de significations.

24 Sur le socle de ces postulats, il est encore possible d'appuyer ce mode de réception ludique de la littérature en ajoutant que, comme avec un jeu, il est envisageable de «tricher » avec un texte : le récepteur peut aller voir à la fin d'un roman policier qui est le meurtrier comme choisir de regarder qui est le coupable d'une partie de Cluedo au lieu de le découvrir grâce à l'enquête comme le prévoient les règles du jeu. Un lecteur peut trouver sur Internet un résumé complet de Pierre et Jean de Maupassant pour identifier $a$ priori les symboles disséminés qui distillent les thèmes du roman dès la première description du port, alors que l'auteur avait probablement prévu que son lecteur modèle les découvre ultérieurement.

Le rapport au jeu prend ici une dimension encore plus ambigüe. La compétition peut en effet être considérée comme marqueur identitaire du jeu. Néanmoins, toujours sur la base des théories d'Umberto Eco, il existe un aspect compétitif dans l'acte de lecture : sommesnous plus «intelligents» que le personnage, le narrateur ou même l'auteur si nous parvenons à démasquer le meurtrier avant le protagoniste principal? La réception s'articule dès lors comme une réaction à un défi ludique. Ce défi n'est possible que grâce à l'incertitude (Baroni, 2007, p. 18) induite par la narrativité et le jeu, encore un trait commun; dans les deux cas, rendre caduque cette incertitude (dans notre exemple du Cluedo ou du roman policier) semble être considéré comme une "tricherie », une rupture du contrat de réception.

Quelques années plus tard, Michel Picard identifie à son tour une spécificité commune cruciale entre espace ludique et littéraire : « Pour n'être pas visuel, pour s'établir à la fois dans le texte, dans le lecteur et dans leur rapport» (Picard, 1986, p. 11). Cet aspect est fondamental : la réception implique la création d'un espace mental, auquel le JdR ajoute la propriété partagée. De surcroît, ce processus ne serait selon lui possible que grâce au soi-disant oxymore entre game et play: "le game discipline le playing, certes, et leur association constitue l'un des caractères fondamentaux de la lecture.» (Picard, 1986, p. 168)

Game et play sont complémentaires. Leur tension fonderait le processus de réception, sorte de chaos systémique et organisé qui caractériserait l'espace mental où se joue l'acte de lecture. Cet espace est sans cesse reconstruit et réactualisé par des mouvements complexes, centrifuges et centripètes, entre le récepteur et l'œuvre. La complexité de ces 
mouvements rend chaque réception d'une même œuvre intrinsèquement unique. Sans aller jusqu'à l'intercréativité, la lecture suppose une part de création propre au récepteur.

\section{Le $J d R$, une étape créative actualisée après la réception?} entreprendre, le système de jeu agissant comme une garantie de cohérence fictionnelle dans ce dispositif conversationnel. En d'autres termes, il s'agit de faire un choix parmi les mondes possibles imaginés par le joueur à un moment précis de la partie, au sens où l'entend Umberto Eco. Comme, en lisant, le lecteur configure ces mondes possibles, en $\mathrm{JdR}$, le joueur imagine de la même manière ces mondes possibles. Le système de jeu est un outil, un guide qui lui permet d'assurer leur intégration au monde actuel de la fiction ${ }^{4}$. Le joueur fait ensuite des choix qui visent à produire le monde qu'il a choisi. Ce monde possible est dès lors actualisé et devient le monde véritable de la fiction, alors que les autres sont désormais écartés.

En ce sens, ce mode de réception aurait tendance à placer les JdR dans la continuité de la lecture selon beaucoup de critiques du $\mathrm{xx}^{\mathrm{e}}$ siècle: "La lecture, en effet, semble la synthèse de la perception et de la création » pour Jean-Paul Sartre (1948, p. 50), elle est « une pratique créatrice, inventive, productrice » pour Roger Chartier (1985, p. 62).

En JdR, le lecteur coopératif d'Umberto Eco devient intercréatif. Le JdR s'inscrit dans le mode de l'unique, de l'exceptionnel, puisqu'aucune partie ne peut être identique à une autre : elle est constituée de créations individuelles. Pendant les parties, la fiction générée par ces créations individuelles l'est uniquement par le biais du langage, comme dans un texte littéraire, les images qui illustrent le matériel de jeu n'étant la plupart du temps qu'accessoires.

31 Nous sommes par conséquent en présence d'un médium que l'on pourrait qualifier de littéraire, d'un point de vue esthétique: "pour l'esthéticien, la littérature se définit comme l'emploi du langage articulé comme matériau de l'œuvre d'art» (Souriau, 1990, p. 955). Cette définition circonscrit le point commun que nous voyons entre littérature et $\mathrm{JdR}$ : le langage permet de produire la matière fictionnelle. La différence, c'est qu'en JdR le langage n'est pas la forme que prend l'œuvre finale (nous verrons que cette œuvre finale n'existe pas, du moins pas sous une forme habituelle), il est un moyen de générer et de réagir à un matériau fictionnel. En d'autres termes, si en littérature le langage est expression à laquelle on apporte un soin esthétique particulier, en JdR c'est avant tout un dispositif de communication et le système de jeu permet d'organiser une conversation pour la rendre productrice d'une substance fictionnelle polyphonique. C'est la raison pour laquelle nous parlons d'intercréativité.

\section{Intercréativité : présentification et oralité, le processus narratif intercréé}

\section{Le présent, temps de la réorganisation narrative}

S'ils disposent tous deux du langage comme matériau de création fictionnelle, contrairement au roman, écrit, figé dans le temps, et produit d'une seule autorité, le JdR exige un rapport conversationnel, oral et présentifié qui lui permet d'atteindre ce statut 
de création collective et plurielle par la langue, comme le relève le créateur de JdR américain Ben Lehman : «Je viens d'une famille d'écrivains ; le langage me fascine. Le JdR est l'un des derniers média qui soit uniquement conversationnel, et les conversations ont leur propre grammaire et leur propre vocabulaire, qui sont différents de ceux de la prose ou de la poésie. » (David, 2015). Le langage est un instrument de pouvoir démiurgique et narratif générant la fiction, et c'est en cela que le JdR, en tant que pratique, peut être rapproché d'une activité littéraire. Étymologiquement, la littérature est rattachée à l'écrit,

mais définir la littérature comme art des seuls écrits méconnaît gravement l'existence d'une importante littérature orale, et d'une culture littéraire réelle, souvent très développée, chez les peuples sans écriture. Pour l'esthéticien, la littérature se définit par l'emploi du langage articulé comme matériau de l'œuvre d'art. [...] C'est un art [la littérature] lié aux organes phonatoires de la voix. Il est ainsi physiologiquement vécu, soit en acte, soit en imagination, soit virtuellement (Souriau, 1990, p. 955-956).

Suivant cette définition, l'emploi du langage serait le matériau qui viendrait constituer l'œuvre. La voix, ou sa projection, serait toujours implicite lors de l'acte de création et de réception littéraire. Le JdR, par l'oral et la présentification, remet la voix au premier plan de la création par ce matériau qu'est le langage, sans avoir besoin de moyens de représentation visuelle, même symboliques, comme des décors, et sans contrainte de l'écrit appris par cœur, comme cela peut être le cas au théâtre.

S'il serait faux de dire que la littérature a totalement oblitéré son caractère oral, ne serait-ce qu'en examinant les expérimentations de l'Oulipo, son rapport à la poésie ou au théâtre, celui-ci demeure tout de même à sa périphérie, que ce soit dans son étymologie (du latin littera, caractère d'un alphabet) dans la manière dont elle est considérée par les non-spécialistes (« les œuvres écrites », nous dit le Grand Robert 2005) ou étudiée.

L'usage du présent sous-tend une redéfinition permanente. Il est la garantie d'une immédiateté indispensable, le «temps réel » qui permet l'échange sur lequel se fonde l'intercréativité. Si le temps intradiégétique peut être sujet aux contractions (ellipses), déplacements (analepses, prolepses) ou extensions (combat au tour par tour), le temps du jeu et de l'édification de l'espace imaginaire commun ne peut que s'inscrire dans l'imminence. Celle-ci permet d'envisager l'éventail des potentialités dont la formulation orale vaut pour une actualisation de l'action dans l'espace diégétique.

Si, en littérature, comme le dit Paul Ricœur (1984, p. 185), le présent de la narration est fictif, en JdR le présent devient un nouveau mode d'élaboration narrative, qui est permis par le jeu. Jeu et narration ont en commun de mettre en scène des conflits. Le JdR modalise ces conflits à l'intérieur d'une narration: le système de jeu répartit le pouvoir sur leur issue, et la fiction la rend signifiante en lui donnant ses enjeux :

Ainsi, beaucoup de récits mettent aux prises, autour d'un enjeu, deux adversaires, dont les "actions » sont de la sorte égalisées; le sujet est alors véritablement double, sans qu'on puisse davantage le réduire par substitution ; c'est même peutêtre là une forme archaïque courante, comme si le récit, à l'instar de certaines langues, avait connu lui aussi un duel de personnes. Ce duel est d'autant plus intéressant qu'il apparente le récit à la structure de certains jeux (fort modernes), dans lesquels deux adversaires égaux désirent conquérir un objet mis en circulation par un arbitre ; ce schéma rappelle la matrice actantielle proposée par Greimas, ce qui ne peut étonner si l'on veut bien se persuader que le jeu, étant un langage, relève lui aussi de la même structure symbolique que l'on retrouve dans la langue et dans le récit : le jeu lui aussi est une phrase (Barthes et alli, 1997, p. 37). 
37 Par le présent, le JdR est le déroulement actif, en temps réel, d'une opposition. Le récit est le déroulement sclérosé parce que passé d'une opposition, sa représentation. L'emploi du présent suppose également le statut homodiégétique du narrateur, « qui suggère presque irrésistiblement une présence du narrateur dans la diégèse » (Genette, 1983, p. 55). Il faut en outre mettre en exergue le rôle du système de jeu dans la présentification du processus narratif, comme l'explique l'auteur de JdR japonais Atsuhiro Okada :

Nous avons beaucoup de moyens qui nous permettent de raconter des histoires (romans, films, jeux vidéo, etc.), mais la plupart nécessitent énormément de temps avant d'arriver jusqu'aux personnes auxquelles ils sont destinés. À l'inverse, un système de jeu de rôle nous permet de laisser s'exprimer notre imagination pendant des heures et de voir l'autre y réagir instantanément (David, 2015, p. 804).

Une fois assimilé, le système de jeu, lorsqu'il ne souffre pas de défauts spécifiques, se pose comme le garant de l'immédiateté du processus d'action et de réaction, capable de s'adapter aux apports créatifs les plus variés. Nous l'avons dit, nous considérons le système de jeu comme l'ensemble des dispositifs qui permet de rendre la fiction interactive et intercréative. En cela il est le cœur de la spécificité médiatique du JdR, puisqu'il engendre une élaboration fictionnelle concomitante lors d'échanges directs. Ces dispositifs fusionnent les processus de création et de réception, c'est en cela qu'Atsuhiro Okada pointe une immédiateté communicationnelle de la fiction. Le présent est le temps de l'improvisation, régulée et soutenue par le système de jeu.

\section{Une transmutation du texte écrit par l'oral}

L'oral est également un trait définitoire des JdR : dès 1997, E. J. Aarseth qualifie les MUD comme une typologie de littérature informatique, OD\&D étant leur ancêtre oral : « les jeux comme Donjons \& Dragons peuvent être considérés comme un cybertexte oral, l'ancêtre oral des jeux vidéo d'aventures textuels. » (Aarseth, 1997, p. 98). Pour autant, cela ne signifie pas que le JdR est détaché de la sphère de l'écrit, bien au contraire. Nous avons évoqué ses origines littéraires et ludiques, mais rappelons que les JdR prennent la forme de livres : règles, tables aléatoires, données encyclopédiques sur l'univers et scénarios sont des textes écrits.

Ce qui est spécifique au JdR, c'est ce rapport alchimique à l'écrit : à partir de l'écrit, donc, le JdR met en place une double transmutation du texte: le MJ (mais cela fonctionne également pour les JdR sans MJ, où ses fonctions sont réparties entre tous les joueurs) transforme une première fois le texte en substrat, en matériau ludique à la lecture des livres du jeu. Ce procédé suppose qu'il va privilégier certaines propriétés du jeu, et en mettre d'autres sous narcose, pour reprendre le terme d'Umberto Eco ${ }^{5}$. Par exemple, un MJ de Shadowrun, après sa lecture, peut davantage être marqué et retenir, consciemment ou pas, plus d'informations concernant le thème de la matrice et l'aspect sociopolitique du jeu (l'intolérance entre les espèces, les inégalités sociales, la malhonnêteté des mégacorporations), car ce sont deux sujets avec lesquels il se sent davantage d'affinités. Cela constitue une première transformation du texte, une réception personnalisante.

Ce substrat extrait, le MJ le transmet aux joueurs qui vont à leur tour le transmuter en jouant, par l'oral, avec tous les mouvements d'intercréativité que cela suppose. En un sens, le JdR est un procédé alchimique du texte écrit vers l'oral, une sorte de processus de «dé-réification». Même les ouvrages de JdR, textes écrits, impulsent cette oralité, 
tendent vers elle et la programment. Il est assez commun que le narrateur s'adresse directement au lecteur dans ces parties à la seconde personne :

Nous avons l'impression que vous avez passé trop de temps à calculer la vélocité d'une balle de $10 \mathrm{~mm}$ à travers du kevlar multicouches. Nous voulons que vous vous détendiez et que vous fassiez du roleplay. Pour nous en assurer, nous avons pensé ce livre pour qu'il soit interactif, à la manière du script d'un film dont vous seriez les acteurs (Ackerman et al., 1993, p. 10).

Dans Castle Falkenstein, dont l'écriture est remarquable, le véritable auteur du jeu, Mike Pondsmith, met en place un dispositif intradiégétique de rédaction. Dès la page 4 , il explique qu'il publie ici, à la demande de son ami Tom, le journal que ce dernier a réussi à lui faire parvenir depuis une dimension parallèle européenne steampunk dans laquelle il a été aspiré. D'emblée, ce mode épistolaire simule là aussi une conversation : «Ce soir j'ai dîné avec un Dragon. Je n'étais pas le plat principal. Je sais que tu ne me croiras pas. Je n'y croirais pas non plus. [...] Mais tu dois m'écouter. Tout ça est bien réel. » (Pondsmith, 1994, p. 5).

44 Ce procédé, qui nous donne à voir le monde en focalisation interne, a pour but de le mettre en tension, de le narrativiser et de jouer avec les attentes du lecteur, toujours sur un mode d'échange : «Pour l'instant, je t'ai raconté comment j'ai été enlevé par magie ; comment j'ai pris part à une quête héroïque pour ramener un roi perdu à ses sujets reconnaissants. [...] Et maintenant, la question à cent mille dollars. Pourquoi ne suis-je pas rentré à la maison?» (Pondsmith, 1994, p. 93). Cette adresse directe au lecteur permet de singulariser le monde et de faire ressentir au récepteur son ambiance, ce qui constituera un outil pour le $\mathrm{MJ}$ : tout au long de sa lecture, celui-ci s'imprégnera de cette atmosphère qu'il devra retransmettre autour de la table.

Cette écriture conversationnelle crée en outre une complicité avec le narrateur qui nous fournit les éléments pour faire jouer au jeu. Il s'accomplit un processus de passation presque solennel de l'œuvre de l'auteur au lecteur-joueur, alors qu'il lui donne les « clés » de l'univers :

Vous trouverez dans ce livre toutes les informations qui vous seront nécessaires pour entreprendre ce fabuleux voyage dans le temps. Nous avons été volontairement ambitieux afin de répondre à toutes les demandes et offrir une vision à la fois globale et détaillée. Nous espérons que cet ensemble d'informations vous permettra de devenir plus intime avec cette époque étonnante. Vos parties de Maléfices y gagneront en richesse, finesse et réalisme. [...] Maléfices tel qu'il a été conçu par ses auteurs est une chose; ce que vous en ferez entre vous en est une autre. Vous n'aurez de ce point de vue aucun compte à rendre, si ce n'est à vos partenaires de jeu (Babarit et alli, 2006, p. 12).

C'est une véritable transmission d'autorité fictionnelle qui s'accomplit par le texte de $\mathrm{JdR}^{6}$ , le lecteur est impliqué et responsabilisé : « Maléfices reste une fiction conçue pour votre plaisir. Il appartient à chacun de définir jusqu'où doit conduire le réalisme historique. » (Babarit et alli, 2006, p. 12). Pour ces raisons, même dans ses textes écrits, le JdR porte les germes de l'oralité qui le caractérise, et qui lui permet de réaliser cette création fictionnelle collective et dynamique. Pour résumer, le présent et l'oralité sont caractéristiques de l'intercréativité permise par le JdR. La fiction co-générée est construite par une conversation. 


\section{Une performance littéraire?}

47 par le langage. Cette expérience dissocie en cela la littérature de l'écrit et propose un nouveau rapport à celle-ci. Avec ce dispositif de communication alternatif qu'est l'intercréativité, les JdR mettent en place une nouvelle manière d'aborder la création fictionnelle par le langage verbal et articulé, comme les jeux vidéo l'ont fait pour la vidéo, alors que le cinéma existait déjà en tant que média vidéo : ce sont deux médias différents qui proposent deux expériences différentes de la fiction en réorganisant le dispositif de communication d'un même moyen, la vidéo.

Le JdR est un processus narratif, une expérience collective et immédiate de la création de la fiction par le langage oral. La fiction est assimilée alors même qu'elle est créée, le phénomène de réception vient nourrir celui de la création. Le JdR est une révolution médiatique dans le sens où il réintègre l'oral et le collectif dans un processus de création fictionnelle. Nous parlons de réintégration, car l'éviction de l'oral de la sphère littéraire 
est somme toute assez récente: "L'information orale, longtemps considérée comme indispensable [...] connut un reflux à mesure que s'accentuait l'emprise de l'écrit. L'historiographie critique de la fin du XIX ${ }^{\mathrm{e}}$ siècle finit par la discréditer complètement » (Boia, 1998, p. 50).

L'écrit comme seul mode valable de la littérature donne à l'auteur un statut d'autorité très marqué : comme le rappellent Isabelle Diu et Élisabeth Parinet (2013), l'auteur n'est pas une posture culturelle innée. Pour elles, si cette figure n'existe pas à l'antiquité, c'est à cause du rapport à l'oral.

Le JdR fait partie de ces points de bascule qui montrent que nous sommes entrés dans le siècle du jeu. Mais au-delà, il semblerait que le mode ludique soit le meilleur chemin de réappropriation populaire de la création fictionnelle, dans une démarche assez similaire à celle qu'a pointée Henry Jenkins (1992) pour les fan fictions. Le JdR est une forme de fiction littéraire qui est allée chercher dans le jeu ce que la forme contemporaine de la littérature, voire de la fiction, interdisait : un processus collectivisé, présentifié et oralisé. Le JdR est donc une prise de pouvoir en cela qu'il annihile les limites entre créateur et récepteur, là où avait été tracée une ligne bien nette. Cette dichotomie relève de la posture sociale, d'une hiérarchie en partie installée par les industries culturelles et les institutions. En JdR, il s'agit non seulement de rétablir cette jouissance de la réception de la fiction littéraire, mais également de la confondre avec la jouissance de la création immédiatement réalisée, transmise et partagée.

La littérature ayant " oublié », dans sa forme actuelle, comment réinstaurer cette égalité, elle a été réinstallée par le jeu. D'un côté, la forme classique de la littérature est écrite, figée et émane d'une seule entité, l'auteur, de l'autre le JdR est oral, présentifié et intercréé. Nous avons ici une littérature apollinienne et une littérature dionysiaque. Cette littérature dionysiaque, refoulée de la culture occidentale à l'ère moderne, ressurgit sous différentes formes : le JdR est une de ses plus importantes incarnations.

\section{BIBLIOGRAPHIE}

AARSETH E. J. (1997), Cybertext: Perspective on Ergodic Literature, Baltimore, Johns Hopkins University Press.

ANDERSON P. (1961), Three Hearts and Three Lions, New York, Doubleday.

BABARIT O., DUGOURD D., GAUDO M., HAMM F. (2006), Maléfices, troisième édition, Cébazat, Éditions du Club Pythagore.

BABCOCK III L. R., CHARRETTE B., DOWD T., HUME P., LEWIS S., WEISMAN J., WYLIE D. (1989), Shadowrun, Chicago, Fasa Corporation.

BAKER V. (2010), Apocalypse World, Lumpley Games.

BARONI R. (2007), La Tension narrative : suspense, curiosité et surprise, Paris, Éditions du Seuil.

BARTHES R., BOOTH W. C., KAYSER W., HAMON P. (1997), Poétique du récit, Paris, Éditions du Seuil. 
BERGSTRÖM K. (2013), « Creativity Rules, how rules impact player creativity in three tabletop roleplaying games », International Journal of Role-Playing, $\mathrm{n}^{\circ}$ 3, pp. 4-17.

BOIA L. (1998), Pour une histoire de l'imaginaire, Paris, Les Belles Lettres.

BOILEAU N. (1998), L’Art poétique, Chant 1 [1674], Paris, Flammarion.

BOOTH W. C., (1961) The Rhetoric of Fiction, Chicago, University of Chicago Press.

BOUCHARDON S. (2008), « Le Récit littéraire interactif : une valeur heuristique », Communication et Langages, $\mathrm{n}^{\circ} 155$, pp. 81-97.

CAILLOIS R. (1957), Les Jeux et les Hommes : le masque et le vertige, Paris, Gallimard.

CHARTIER R. (1985), « Du livre au livre », in Chartier R. (dir.), Pratiques de la lecture, Marseille, Éditions Rivages.

DAVID C. (2015), Jeux de rôle sur table : l'intercréativité de la fiction littéraire, thèse de doctorat, Université de Paris XIII.

DE MAUPASSANT (1999) G., Pierre et Jean [1887], Paris, Gallimard.

DIU I., PARINET É. (2013), Histoire des auteurs, Paris, Éditions Perrin.

DUFLO C. (1997), Jouer et philosopher, Paris, PUF.

DUVIGNAUD J. (1980), Le Jeu du jeu, Paris, Éditions Balland.

ECO U. (1993), Lector in fabula [1979] Paris, Le Livre de Poche.

GENETTE G. (1972), Figures III, Paris, Éditions du Seuil.

GENETTE G. (1983), Nouveau discours du récit, Paris, Éditions du Seuil.

GORDEN Gr., KERN R., KLUG G. C., RANDALL N. (1983), James Bond 007, New York, Victory Games.

HAMMER J. (2007), « Agency and Authority in Role-Playing Texts », in Knobel M., Lankshear C. (dir.), A New Literacies Sampler, New York, Lang Press, pp. 67-94.

HENRIOT J. (1989), Sous couleur de jouer : la métaphore ludique, Paris, José Corti Éditions.

HENRY L. (2003), Group Narration: Power, Information, and Play in Role Playing Games, http:// darkshire.net/jhkim/rpg/theory/liz-paper-2003

HOGGART R. (1970), La Culture du pauvre, Paris, Les Éditions de Minuit.

ISER W. (1995), L'Acte de lecture, théorie de l'effet esthétique, Bruxelles, Éditions Mardaga.

JENKINS H. (1992), Textual Poachers: Television Fans and Participatory Culture, New York, Routledge.

KLEINERT T. W. (2006), « Jazzing it up: Improvisation and Thematic Play », in Walton J. (dir.), Push, New Thinking about Roleplaying, bleedingplay.wordpress.com/push/

LAVOCAT F. (2005), « Fiction et paradoxes : les nouveaux mondes possibles à la Renaissance », in Lavocat $\mathrm{F}$. (dir.), Usages et théories de la fiction, le débat contemporain à l'épreuve des textes anciens (XVI -XVIII ${ }^{e}$ siècles), Rennes, Presses Universitaires de Rennes.

MACKAY D. (2001), The Fantasy Role-Playing Game, a New Performing Art, McFarland, Jefferson.

MOLLIER J. -Y. (2001), La Lecture et ses publics à l'époque contemporaine. Essais d'histoire culturelle, Paris, PUF.

MONTFORT N. (2005), Twisty Little Passages, an Approach of Interactive Fiction, Cambridge, MIT Press. 
PICARD M. (1986), La Lecture comme jeu, Paris, Les Éditions de Minuit.

PONDSMITH M. A. (1994), Castle Falkenstein, Berkeley, R. Talsorian Games.RIC@UR P. (1984), Temps et récit, 2. La configuration dans le récit de fiction, Paris, Seuil.

RYAN M.-L. (2003), Narrative as Virtual Reality: Immersion and Interactivity in Literature and Electronic Media, Baltimore, Johns Hopkins University Press.

SARTRE J.-P. (1948), Qu'est-ce que la littérature ? Paris, Gallimard.

SCHAEFFER J.-M. (1999), Pourquoi la fiction? Paris, Éditions du Seuil.

SINTES F. (2009), La Rédaction d'un jeu influe-t-elle sur sa pratique? www.limbicsystemsjdr.com/laredaction-dun-jeu-influe-t-elle-sur-sa-pratique/

SOURIAU É. (1990), Vocabulaire d'esthétique, Paris, PUF.

TOLKIEN J.R.R. (1954), The Lord of the Rings, Londres, Allen \& Uwin.

VANCE J. (1950), The Dying Earth, New York, Hillman Periodicals.

WINNICOTT D. (2002), Jeu et réalité, [1975], Paris, Gallimard.

\section{NOTES}

1. Dans le cas des JdR dits traditionnels qui comprennent un meneur de jeu (MJ), l'autorité générative lui est généralement en grande partie dévolue (bien que ceci ait évolué, par exemple dans Apocalypse World où le $\mathrm{MJ}$ définit des oppositions "fronts ", mais où le reste du monde est imaginé pour les joueurs), et l'autorité résolutive au système de résolution, qui comporte habituellement un facteur aléatoire (jet de dés), bien que certaines règles comme celles de James Bond 007 transmettent une partie de cette autorité aux joueurs avec les points d'héroïsme.

2. Jeu de société de stratégie, qui simule des batailles entre armées. Le JdR en a notamment hérité le fait de considérer les règles comme des guides plutôt que des lois, comme il est impossible de prévoir une règle précise pour chaque situation.

3. Soit « l'univers spatio-temporel désigné par le récit » (Genette, 1972, p. 48).

4. Les règles peuvent également renforcer le sentiment de cohérence fictionnelle lorsque les participants jouent divers modules (aventures déjà écrites vendues pour un jeu donné) prenant place dans un même univers qui possède sa propre historicité fictive, mais dans différents lieux et époques.

5. «Quand il découvre chaque terme, le lecteur privilégie certaines propriétés et en garde d'autres sous narcose » (Eco, 1979, p. 109).

6. Jessica Hammer (2007) distingue trois niveaux d'auteurs en JdR. Le premier comprend ceux qui ont fait l'univers et le système. Le deuxième compte le créateur du scénario. Le troisième est celui du texte qui émerge lors de la partie. «Le troisième auteur "écrit" le texte du jeu en jouant. »

7. Car l'improvisation théâtrale répond à une logique similaire: «Son [l'improvisation] plus grand mérite est de permettre l'identification totale du comédien à son personnage ; en ce sens elle est une expérience qui ne peut être qu'unique » (Souriau, 1990, p. 874).

8. Il n'est ainsi pas rare d'observer des analogies avec le théâtre d'improvisation et la musique improvisée : le lien entre jazz et JdR est évoqué par Timothy Walters Kleinert (Walton, 2006), par Frédéric Sintes (2009), par Catharine Russell McAskill (Henry, 2003), Karl Bergström (2013, p. 5), ou notre thèse (David, 2015).

9. Pour Jacques Henriot (1989, p. 155), l'attitude ludique c'est « l'imaginaire en acte ». 
10. «Un texte est émis pour quelqu'un capable de l'actualiser - même si on n'espère pas (ou ne veut pas) que ce quelqu'un existe concrètement ou empiriquement » (Eco, 1979, p. 64).

11. Au sens de « signifiant, énoncé, discours ou texte narratif » (Genette, 1972, p. 72).

12. «L'acte narratif producteur et, par extension, l'ensemble de la situation réelle ou fictive dans laquelle il prend place» (Genette, 1972, p. 72).

13. « Le signifié ou le contenu narratif.» (Genette, 1972, p. 72).

\section{RÉSUMÉS}

Cet article traite du jeu de rôle sur table comme moyen oral et collectif de création fictionnelle par le langage verbal, et interroge son rapport au littéraire. Il explique la manière dont les jeux de rôle mettent en place des dispositifs ludiques qui permettent, voire obligent, les joueurs à cogénérer un matériau fictionnel démiurgique et narratif, selon le principe qui est nommé l'intercréativité. Ainsi, les jeux de rôle font de la réception une étape de création de la fiction. Mais peuvent-ils être considérés comme une forme de jeu littéraire oral et collectif? Pour répondre à cette question, cet article analyse dans un premier temps le rapport qu'entretiennent littérature et jeu d'une façon générale. Ensuite, il étudie le $\mathrm{JdR}$ en tant que processus conversationnel, oral et "présentifié » qui génère une création fictionnelle collective. Avec l'intercréativité, c'est un rapport dionysiaque à la littérature qui ressurgit, alors qu'il avait été annihilé à l'ère moderne.

This article deals with pen \& paper role-playing games as an oral and collective way to create fiction through verbal language, and questions its relation to literature. It explains role-playing games set up game procedures to enable, even oblige, players to co-create narrative and diegetic contents, according to what we call intercreativity. Thus, role-playing games turn readerresponse into a step of fictional creation. But can they be considered as a form of oral and collective literary game? To answer this question, this article first analyzes the relation between literature and games. Then, it examines role-playing games as a conversational, oral and "presentified" process generating collective and fictional creation. Intercreativity makes reappear a Dionysian way to consider literature, which has been annihilated during the Modern era.

\section{INDEX}

Mots-clés : jeux de rôle, littérature, interactivité, intercréativité, réception, littérature orale, fiction, narration

Keywords : roleplaying game, literature, interactivity, intercreativity, oral literature, narrative

\section{AUTEUR}

CORALIE DAVID

Université Paris 13 - Sorbonne Paris Cité 